Proyecciones Journal of Mathematics

Vol. 33, $\mathrm{N}^{\circ}$ 4, pp. 437-446, December 2014.

Universidad Católica del Norte

Antofagasta - Chile

\title{
Difference sequence spaces in cone metric space
}

\author{
Binod Chandra Tripathy \\ Institute of Advanced Study in Science and Tech., India \\ Nanda Ram Das \\ and \\ Rinku Dey \\ Gauhati University, India \\ Received: July 2014. Accepted: July 2014
}

\begin{abstract}
In this article we introduce the notion of difference bounded, convergent and null sequences in cone metric space. We investigate their different algebraic and topological properties.
\end{abstract}

Keywords and phrases : Cone metric, Complete metric space, Difference sequence, Solid space, Symmetric space.

AMS Classification : 40A05, 46B20, 54E35 


\section{Introduction}

The notion of difference sequence spaces (for single sequences) was introduced by Kizmaz [4] as follows:

$$
Z(\Delta)=\left\{\left(x_{k}\right) \in w:\left(\Delta x_{k}\right) \in Z\right\}
$$

for $Z=c, c_{0}$ and $\ell_{\infty}$, where $\Delta x_{k}=x_{k}-x_{k+1}$ for all $k \in N$.

The above spaces are Banach spaces normed by

$$
\left\|\left(x_{k}\right)\right\|=\left|x_{1}\right|+\sup _{k \geq 1}\left|\Delta x_{k}\right| .
$$

Later on the notion of difference sequences was investigated from different aspects by Tripathy et al [5], Tripathy and Baruah [6], Tripathy and Borgogain [8], Tripathy and Chandra [9], Tripathy and Debnath [10], Tripathy and Dutta [12], Tripathy and Goswami [12] and many others.

The notion of cone metric space has been applied by various authors in different fields of research in these days. It has been applied for introducing and investigating different new classes of sequence spaces and studying their different algebraic and topological properties by Abdeljawad [1], Beg, Abbas and Nazir [2], Dhanorkar and Salunke [3] and many others. In this article we have investigated different properties of the notion of difference bounded, convergent and null sequences in cone metric spaces.

\section{Definitions and Preliminaries}

Definition 2.1. A subset $P$ of a real Banach space $E$ is called a cone if and only if

(i) $P$ is closed, non-empty and $P \neq\{0\}$.

(ii) If $a, b \in R, a \geq 0, b \geq 0$ and $x, y \in P$, then $a x+b y \in P$.

(iii) If both $x \in P$ and $-x \in P$ then $x=0$.

For a given cone $P \subseteq E$, we can define a partial ordering $\leq$ with respect to $P$ by $x \leq y$ if and only if $y-x \in P, x<y$ will stand for $x \leq y$ and 
$x \neq y$, while $x<<y$ will stand for $y-x \in$ int $P$, where int $P$ denotes the interior of $P$.

Definition 2.2. A cone metric space is an ordered pair $(X, d)$, where $X$ is any set and $d: X \times X \rightarrow E$ is a mapping satisfying:

(i) $0<d(x, y)$ for all $x, y \in X$.

(ii) $d(x, y)=0$ if and only if $x=y$.

(iii) $d(x, y)=d(y, x)$ for all $x, y \in X$.

(iv) $d(x, y) \leq d(x, z)+d(z, y)$ for all $x, y, z \in X$.

Example 2.1. Let $E=R^{2}, P=\{(x, y) \in E: x, y \geq 0\}, X=R$ and $d: X \times X \rightarrow E$ defined by $d(x, y)=(|x-y|, \alpha|x-y|)$, where $\alpha \geq 0$, is a constant and $x, y \in X$. Then it is well known that $(X, d)$ is a cone metric space.

Example 2.2. Let $E$ be a Banach space, $P=\{(x, y, z) \in E: x, y, z \geq 0\}$ and $(X, \eta)$ be a metric space with $\theta$ the zero element. Let $d: X \times X \rightarrow E$ be defined by $d(x, y)=(\eta(x, y), \alpha \eta(x, y), \beta \eta(x, y)), \alpha, \beta \geq 0$.

Then it can be easily verified that $(X, d)$ is a cone metric space.

Definition 2.3. A sequence space $E$ is said to be solid or normal if $\left\{\alpha_{k} x_{k}\right\} \in E$ whenever $\left\{x_{k}\right\} \in E$ and for all sequences $\left(\alpha_{k}\right)$ of scalars with $\left|\alpha_{k}\right| \leq 1$ for all $k \in N$.

Let $K=\left\{k_{1}<k_{2}<k_{3}, \ldots\right\} \subseteq N$ and $E$ be a class of sequences. A $K$-step set of $E$ is a set of sequences $\lambda_{K}^{E}=\left\{\left(x_{k_{n}}\right) \in w:\left(x_{k}\right) \in w\right\}$.

A canonical pre-image of a sequence $\left(x_{k_{n}}\right) \in \lambda_{K}^{E}$ is a sequence $\left(y_{n}\right) \in$ $w$, defined as follows:

$$
y_{n}= \begin{cases}x_{n} & \text { if } n \in K \\ \overline{0}, & \text { otherwise }\end{cases}
$$

where $\overline{0}$ is the zero element. 
Definition 2.4. A canonical pre-image of a step set $\lambda_{K}^{E}$ is a set of canonical pre-images of all elements in $\lambda_{K}^{E}$ i.e. $Y$ is in canonical pre-image $\lambda_{K}^{E}$ if and only if $Y$ is canonical pre-image of some $X \in \lambda_{K}^{E}$.

Definition 2.5. A class of sequences $E$ is said to be monotone if $E$ contains the canonical pre-images of all its step sets.

The following remark is well known.

Remark 2.1. A class of sequences $E$ is solid $\Rightarrow E$ is monotone.

Definition 2.6. A sequence space $E$ is said to be convergence free if $\left\{x_{n}\right\} \in E$ implies $\left\{y_{n}\right\} \in E$ such that $y_{n}=\overline{0}$, whenever $x_{n}=\overline{0}$.

Definition 2.7. A class of sequences $E$ is said to be symmetric if $\left(x_{\pi(n)}\right) \in$ $E$, whenever $\left(x_{k}\right) \in E$, where $\pi$ is a permutation of $N$.

Definition 2.8. A sequence space $E$ is said to be a sequence algebra if $\left(x_{k} y_{k}\right) \in E$, whenever $\left(x_{k}\right),\left(y_{k}\right) \in E$.

In this article we introduced the following definitions.

Definition 2.9. Let $(X, d)$ be a cone metric space. A sequence $\left(x_{k}\right)$ in $c(\Delta)$ is said to be convergent to $x$ if for every $\bar{c} \in E$ with $\theta<<\bar{c}$, there exists $n_{0}$ such that for all $k \geq n_{0}, d\left(\Delta x_{k}, x\right)<<\bar{c}$.

Definition 2.10. Let $(X, d)$ be a cone metric space. A sequence $\left(x_{k}\right)$ in $c(\Delta)$ is said to be Cauchy sequence if for every $\bar{c} \in E$ with $\theta<<\bar{c}$ there exists $n_{0}$ such that for all $k, p \geq n_{0}, d\left(\Delta x_{k}, \Delta x_{p}\right)<<\bar{c}$, where $\bar{c}=(c, \alpha c, \beta c), \alpha, \beta \geq 0$.

We procure the following results those will be used in establishing results of this article.

Lemma 2.1. Let $(X, d)$ be a cone metric space and $\left(x_{k}\right),\left(y_{k}\right)$ be in $c(\Delta)$. If $\lim _{k \rightarrow \infty} \Delta x_{k}=x$ and $\lim _{k \rightarrow \infty} \Delta y_{k}=y$, then $\lim _{k \rightarrow \infty} d\left(\Delta x_{k}, \Delta y_{k}\right)=d(x, y)$.

Lemma 2.2. Let $(X, d)$ be a cone metric space and $\left(x_{k}\right) \in c(\Delta)$. If 
$\lim _{k \rightarrow \infty} \Delta x_{k}=x$ and $\lim _{k \rightarrow \infty} \Delta x_{k}=y$, then $x=y$.

Lemma 2.3. Let $(X, d)$ be a cone metric space and $\left(x_{k}\right) \in c(\Delta)$. If $\lim _{k \rightarrow \infty} \Delta x_{k}=x$, then $d\left(\Delta x_{k}, x\right)=0$.

\section{Main results}

We state the following result without proof.

Theorem 3.1. $(Z(\Delta), d)$, for $Z=c, c_{0}, \ell_{\infty}$ are cone metric spaces.

Theorem 3.2. Let $x$ be a complete cone metric space. Then the classes of sequences $c(\Delta), c_{0}(\Delta), \ell_{\infty}(\Delta)$ are complete cone metric spaces w.r.t. the cone metric $\rho(x, y)=d\left(x_{1}, y_{1}\right)+\sup _{k \in N} d\left(\Delta x_{k}, \Delta y_{k}\right)$.

Proof. Let $\left(x^{(i)}\right)$ be a Cauchy sequence in $\ell_{\infty}(\Delta)$.

Then for a given $\bar{c} \in E$ with $\bar{c}=(c, \alpha c, \beta c)$ there exists $n_{0}$ such that

$\rho\left(x^{i}, x^{j}\right)<<\bar{c}$ for all $i, j \geq n_{0}$.

$\Rightarrow d\left(x_{1}^{i}, x_{1}^{j}\right)+\operatorname{Sup}_{k \in N} d\left(\Delta x_{k}^{i}, \Delta x_{k}^{j}\right)<<\bar{c}$.

$\Rightarrow d\left(x_{1}^{i}, x_{1}^{j}\right)<<\bar{c}$ and $d\left(\Delta x_{k}^{i}, \Delta x_{k}^{j}\right)<<\bar{c}$, for all $k \in N$.

$\Rightarrow\left(\eta\left(x_{k}^{i}, x_{k}^{j}\right), \alpha \eta\left(x_{k}^{i}, x_{k}^{j}\right), \beta \eta\left(x_{k}^{i}, x_{k}^{j}\right)\right)<<(c, \alpha c, \beta c)$

and $\left(\eta\left(\Delta x_{k}^{i}, \Delta x_{k}^{j}\right), \alpha \eta\left(\Delta x_{k}^{i}, \Delta x_{k}^{j}\right), \beta \eta\left(\Delta x_{k}^{i}, \Delta x_{k}^{j}\right)\right)<<(c, \alpha c, \beta c)$.

$\Rightarrow \eta\left(x_{k}^{i}, x_{k}^{j}\right)<<c$ and $\eta\left(\Delta x_{k}^{i}, \Delta x_{k}^{j}\right)<<c$.

Now, $\eta\left(x_{k}^{i}, x_{k}^{j}\right)<<c$.

$\Rightarrow\left(x_{1}^{i}\right)$ is a Cauchy sequence in $X$.

Since $X$ is a complete cone metric space, so $\left(x_{1}^{i}\right)$ converges to $L$ in $X$. 


$$
\Rightarrow d\left(x_{1}^{i}, L_{1}\right)<<\frac{\bar{c}}{2}
$$

and $\eta\left(\Delta x_{k}^{i}, \Delta x_{k}^{j}\right)<<c$.

$\Rightarrow \eta\left(x_{k}^{i}-x_{k+1}^{i}, x_{k}^{j}-x_{k+1}^{j}\right)<<c$.

$\Rightarrow \eta\left(x_{t}^{i}, x_{t}^{j}\right)<<c$, where $x_{t}^{i}=x_{k}^{i}-x_{k+1}^{i}$ and $x_{t}^{j}=x_{k}^{j}-x_{k+1}^{j}$.

$\Rightarrow\left(x_{t}^{i}\right)$ is a Cauchy sequence in $X$.

Since $X$ is complete, so $\left(x_{k}^{i}\right)$ converges to $L_{k}$, for each $k \in N$.

Therefore $d\left(x_{k}^{i}, L_{k}\right)<<c$, for all $k \in N$.

$\Rightarrow d\left(\Delta x_{k}^{i}, L_{k}\right)<<\bar{c}$, for all $k \in N$.

$$
\Rightarrow \sup _{k \in N} d\left(\Delta x_{k}^{i}, L_{k}\right)<<\frac{\bar{c}}{2}
$$

Adding (3.1) and (3.2) we get,

$$
\begin{aligned}
& d\left(x_{1}^{i}, L_{1}\right)+\sup _{k \in N} d\left(\Delta x^{i}{ }_{k}, L_{k}\right)<<\bar{c} . \\
\Rightarrow & d\left(x^{i}, L\right)<<\bar{c} . \\
\Rightarrow & \left(x^{i}\right) \text { converges to } L .
\end{aligned}
$$

Hence $\ell_{\infty}(\Delta)$ is a complete metric space.

Theorem 3.3. The class of all sequences $c(\Delta), c_{0}(\Delta), \ell_{\infty}(\Delta)$ are neither solid nor normal.

Proof. The result can be verified by the following example.

Example 3.1. Let us consider the cases $c(\Delta)$ and $c_{0}(\Delta)$, similar example can be constructed for the case $\ell_{\infty}(\Delta)$. Let $E=R^{3}, P=(x, y, z) \in E: x, y, z \geq 0, X=$ $R, d: X \times X \rightarrow E$ be defined by 


$$
d(x, y)=(|x-y|, \alpha|x-y|, \beta|x-y|),
$$

where $\alpha, \beta \geq 0$ are constants. Consider the sequence $\left(x_{k}\right)$ defined by $x_{k}=1$, for all $k \in N$.

Then $\Delta x_{k}=0$, for all $k \in N$.

Then clearly $\left(x_{k}\right) \in c(\Delta)$ is convergent to 0 with respect to the cone metric space considered.

We have $\left(\Delta x_{k}\right)=(0)$ is convergent.

Consider the canonical pre-image of $\left(x_{k}\right)$ defined by

$\left(y_{k}\right)=\left(x_{1}, 0, x_{3}, 0, x_{5}, 0, \ldots\right)=(1,0,1,0,1,0, \ldots)$.

Now consider the sequence of scalars $\left(\alpha_{k}\right)$ defined by $\alpha_{k}=(-1)^{k}$, for all $k \in N$.

Then it can be easily verified that the sequence $\Delta\left(\alpha_{k} x_{k}\right)=\Delta\left((-1)^{k} x_{k}\right)$ is not convergent with respect to the cone metric consider above.

Then $\left(\Delta x_{k}\right)=(1,-1,1,-1,1,-1, \ldots) \notin c(\Delta)$.

Hence the class of all convergent sequences is not normal and hence is not solid.

Theorem 3.4. The class of all sequences $c(\Delta), c_{0}(\Delta), \ell_{\infty}(\Delta)$ are not symmetric.

Proof. The result can be verified by the following example.

Example 3.2. Consider the cone metric space considered in Example 3.1. Consider the sequence $\left(x_{k}\right)$ defined by

$$
\left(x_{k}\right)=(1,2,3,4, \ldots, k, \ldots) .
$$

Then $\left(\Delta x_{k}\right)=(-1,-1,-1, \ldots)$. 
Hence $\left(\Delta x_{k}\right)$ converges with respect to the cone metric space considered.

Considering the rearrangement $\left(y_{k}\right)$ of $\left(x_{k}\right)$ defined by $\left(y_{k}\right)=(1,2,4,3,9,5,16,6,25,7, \ldots)$.

$\left(\Delta y_{k}\right)=(-1,-2,1,-6,4,-11,10, \ldots)$

Then it can be easily examined that the sequence $\left(\Delta y_{k}\right)$ is not convergent with respect to the cone metric consider above.

Hence the class of all sequences $c(\Delta), c_{0}(\Delta), \ell_{\infty}(\Delta)$ are not symmetric.

Theorem 3.5. The class of all sequences $c(\Delta), c_{0}(\Delta), \ell_{\infty}(\Delta)$ are not sequence algebra.

Proof. The result can be verified by the following example.

Example 3.3. Consider the cone metric space considered in Example 3.1. Consider the sequence $\left(x_{k}\right)$ and $\left(y_{k}\right)$ defined by

$x_{k}=y_{k}=k$, for all $k \in N$.

Then $\Delta x_{k}=\Delta y_{k}=-1$, for all $k \in N$.

Therefore $\left(x_{k}\right),\left(y_{k}\right) \in c(\Delta)$.

Next we have $\Delta\left(x_{k} y_{k}\right)=k^{2}-(k+1)^{2}=-2 k-1$, for all $k \in N$.

Hence $d\left(\Delta\left(x_{k} y_{k}\right), \overline{0}\right) \rightarrow(\infty, \infty, \infty)$.

So the class of all sequences $c(\Delta), c_{0}(\Delta), \ell_{\infty}(\Delta)$ are not sequence algebra.

\section{References}

[1] T. Abdeljawad, Completion of cone metric spaces, Hacettepe Jour. Math. Stat., 39 (1), pp. 67-74, (2010). 
[2] I. Beg, M. Abbas and T. Nazir, Generalized cone metric space, J. Nonlinear Sci. Appl., 3 (1), pp. 21-31, (2010)).

[3] G. A. Dhanorkar and J. N. Salunke, A Generalization on Fixed Point Theorem on Cone Metric Spaces with $\omega$-Distance, Internat. Math. Forum, 6(39), pp. 1915-1919, (2011).

[4] H. Kizmaz, On certain sequence spaces, Canad. Math. Bull., 24, pp. 169-176, (1981).

[5] B. C. Tripathy, Y. Altin and M. Et, Generalized difference sequences spaces on seminormed spaces defined by Orlicz functions, Math. Slovaca, 58 (3), pp. 315-324, (2008).

[6] B. C. Tripathy and A. Baruah, Lacunary statistically convergent and lacunary strongly convergent generalized difference sequences of fuzzy real numbers, Kyungpook Math. J., 50 (4), pp. 565-574, (2010).

[7] B. C. Tripathy and A. Baruah, M.Et, M. Gungor, On almost statistical convergence of new type of generalized difference sequence of fuzzy numbers, Iranian Jour. Sci. Tech, Trans. A; Sci., 36 (2), pp. 147-155, (2012).

[8] B. C. Tripathy and S. Borgogain, Some classes of difference sequence spaces of fuzzy real numbers defined by Orlicz function, Advances in Fuzzy Systems, 2011, Article ID216414, 6 pages.

[9] B. C. Tripathy and P. Chandra, On some generalized difference paranormed sequence spaces associated with multiplier sequences defined by modulus function, Anal. Theory Appl., 27 (1), pp. 21-27, (2011).

[10] B. C. Tripathy and S. Debnath, On generalized difference sequence spaces of fuzzy numbers, Acta Scientiarum. Technology, 35 (1), pp. 117-121, (2013).

[11] B. C. Tripathy and H. Dutta, On some new paranormed difference sequence spaces defined by Orlicz functions, Kyungpook Math J., 50 (1), pp. 59-69, (2010).

[12] B. C. Tripathy and R. Goswami, On triple difference sequences of real numbers in probabilistic normed spaces, Proyecciones Jour. Math., 33 (2), pp. 157-174, (2014). 


\section{Binod Chandra Tripathy}

Mathematical Sciences Division

Institute of Advanced Study in cience and Technology

Paschim Boragaon; Garchuk

Guwahati - 781035,

Assam

India

e-mail : tripathybc@rediffmail.com

\section{Nanda Ram Das}

Department of Mathematics

Gauhati University

Guwahati - 781014,

Assam

India

e-mail : rinkudey_math@rediffmail.com

and

\section{Rinku Dey}

Department of Mathematics

Gauhati University

Guwahati - 781014,

Assam

India

e-mail : nrd47@yahoo.co.in 